Research Paper

\title{
CDK5 and Its Activator P35 in Normal Pituitary and in Pituitary Adenomas: Relationship to VEGF Expression
}

\author{
Weiyan Xie, Hongyun Wang, Yue He, Dan Li, Lei Gong, Yazhuo Zhang ${ }^{\bowtie}$ \\ Beijing Neurosurgical Institute, Beijing Tiantan Hospital Affiliated to Capital Medical University, Beijing 100050, China.
}

$\triangle$ Corresponding author: Ya-Zhuo Zhang, Beijing Neurosurgical Institute, Beijing Tiantan Hospital Affiliated to Capital Medical University, Beijing 100050, China (Tel: +86-10-67022886 Fax: +86-10-67057391, Email:zyz2004520@163.com).

(ㅇ Ivyspring International Publisher. This is an open-access article distributed under the terms of the Creative Commons License (http://creativecommons.org/ licenses/by-nc-nd/3.0/). Reproduction is permitted for personal, noncommercial use, provided that the article is in whole, unmodified, and properly cited.

Received: 2013.09.27; Accepted: 2014.01.17; Published: 2014.01.25

\begin{abstract}
Pituitary tumors are monoclonal adenomas that account for about 10-15\% of intracranial tumors. Cyclin-dependent kinase 5 (CDK5) regulates the activities of various proteins and cellular processes in the nervous system, but its potential roles in pituitary adenomas are poorly understood. The kinase activity of CDK5 requires association with an activating protein, $\mathrm{p} 35$ (also known as CDK5 activator I, p35). Here, we show that functional CDK5, associated with $\mathrm{p} 35$, is present in normal human pituitary and in pituitary tumors. Furthermore, p 35 mRNA and protein levels were higher in pituitary adenomas than in the normal glands, suggesting that CDK5 activity might be upregulated in pituitary tumors. Inhibition of CDK5 activity in rat pituitary cells, reduced the expression of vascular endothelial growth factor (VEGF), a protein that regulates vasculogenesis and angiogenesis. Our results suggest that increased CDK5-mediated VEGF expression might play a crucial role in the development of pituitary adenomas, and that roscovitine and other CDK5 inhibitors could be useful as anticancer agents.
\end{abstract}

Key words: Cdk5; p35; VEGF; pituitary adenomas; expression.

\section{Introduction}

Pituitary tumors comprise $10-25 \%$ of all intracranial tumors. Patients with pituitary tumors often suffer from serious disorders affecting development, growth, fertility or the ability to cope with labor, because of dysfunctional production of hormones. Pituitary tumors derive from different cell types of the anterior pituitary. Tumors are classified clinically according to hormonal status into functioning and nonfunctioning adenomas. Some of these tumors, known as functional adenomas, still produce pituitary trophic hormones (often in excessive amounts) such as prolactin (PRL, $25-41 \%$,), growth hormone $(\mathrm{GH}$, 10-20\%), adrenocorticotrophic hormone (ACTH, $5-15 \%)$ or, rarely, luteinizing hormone (LH) or follicle-stimulating hormone (FSH). These hypersecretory activities are associated with hypogonadism, infertility, acromegaly, Cushing's disease and hyperthyroidism $(17,18)$. Previous studies of pituitary tumors have identified several mutations that, by activating oncogenes or inactivating tumor suppressors, could be involved in tumorigenesis (30). However, a general mechanism for the origin of pituitary tumors remains obscure.

Pathological angiogenesis is important for tumor growth because it promotes oxygenation, nutrient perfusion and removal of metabolic waste. In contrast to the situation in other tissues, vascularization is lower in pituitary adenomas than in the normal gland. However, increased vascularity seems to be related to different aspects of prolactinoma behavior including size, invasiveness, surgical outcome and malignancy (28).

Vascular endothelial growth factor (VEGF) plays a crucial role in vasculogenesis and angiogenesis not only during development but also during pathological processes, by stimulating proliferation and migration of endothelial cells (5) and by inducing fenestrations in the endothelium, leading to increased endo- 
thelial permeability. About $90 \%$ of human pituitary tumors show detectable VEGF secretion in vitro (15, 24). VEGF expression can also be measured by immunohistochemistry and in situ hybridization in pituitary adenomas $(14,19)$. VEGF and its receptors are widely expressed in normal and adenomatous human pituitary, suggesting a role for VEGF in promoting pituitary cell proliferation $(20,21)$. Moreover, administration of VEGF-blocking antibodies inhibits growth of pituitary adenomas in endocrine neoplasia type 1 . These findings suggest a role for VEGF in the modulation of pituitary tumor angiogenesis, which could be part of a mechanism by which estrogens cause pituitary hyperplasia and possibly prolactinoma formation.

The CDK5-p35 complex plays a key role in the development and growth of the nervous system. In addition, CDK5 and another activating protein, p39 (also known as CDK5 activator 2), are expressed in rat anterior pituitary and affect pituitary hormone exocytosis and cortical actin organization (29). CDK5-mediated phosphorylation of EPRS (bifunctional glutamate/proline-tRNA ligase) regulates VEGF-A expression in U937 monocytes (2), indicating that CDK5 might be involved in VEGF-A expression. Therefore, investigating CDK5 targets may shed light on the mechanisms of pituitary tumor formation.

In the present study, we set out to elucidate the role of CDK5 in human pituitaries and various types of pituitary adenomas by examining the expression and cellular localization of CDK5 and p35. The expression of both proteins in the two tissue types was confirmed at the protein and mRNA levels by western blot, immunohistochemical staining and by RT-PCR, respectively. Upregulation of p35, but not of CDK5, was observed in pituitary adenomas. Moreover, we found that inhibition of CDK5 activity reduced VEGF expression in a rat pituitary cell line (GH3 cell line). Taken together, the results suggest that pituitary adenomas might be functionally regulated by CDK5 through its effects on VEGF expression, opening new research avenues for drug development and for control of pituitary adenomas.

\section{Materials and Methods}

\section{Tissue specimens}

Human pituitary adenomas were obtained after their surgical resection from consecutive unselected patients who underwent pituitary surgery at Beijing Tiantan Hospital. Portions of the surgical specimen were immediately frozen and stored in liquid nitrogen. As controls, 8 normal pituitary glands were obtained from adults who had died of accidents and were also studied within 12 hours of death. Informed consent was obtained from all patients, and the study was approved by the local Ethics Committee. A list of the 18 pituitary adenomas included in the study and their clinical diagnosis are given in Table 1 . They were classified into PRL-secreting adenomas (7 cases), GH-secreting adenomas (3 cases), nonfunctioning pituitary adenomas (8 cases). Tumors were graded according to a modified Hardy's classification (23).

Table I. Clinical characteristics in 18 pituitary adenomas.

\begin{tabular}{|c|c|c|c|c|c|c|}
\hline $\begin{array}{l}\text { Patient } \\
\text { no. }\end{array}$ & $\begin{array}{l}\text { Age } \\
\text { (yr) }\end{array}$ & Sex & $\begin{array}{l}\text { Clinical } \\
\text { diagnosis }\end{array}$ & $\begin{array}{l}\text { Ki-67 } \\
(\%)\end{array}$ & $\begin{array}{l}\text { Tumor size } \\
(\mathrm{mm})\end{array}$ & Grade \\
\hline 1 & 46 & $\mathrm{M}$ & NFPA & 2 & 1.8 & I \\
\hline 2 & 46 & F & $\mathrm{GH}$ & 0.2 & 0.7 & I \\
\hline 3 & 59 & F & NFPA & 0.9 & 3.1 & III \\
\hline 4 & 52 & M & PRO & 0.3 & 2.6 & III \\
\hline 5 & 53 & M & $\mathrm{GH}$ & 2.8 & 1.7 & II \\
\hline 6 & 51 & M & NFPA & 4 & 1.9 & II \\
\hline 7 & 51 & F & PRO & 0.4 & 2 & II \\
\hline 8 & 37 & F & PRO & 0.2 & 0.9 & I \\
\hline 9 & 34 & F & NFPA & 1.2 & 3.2 & III \\
\hline 10 & 47 & M & NFPA & 0.5 & 3.3 & III \\
\hline 11 & 46 & F & NFPA & 1.7 & 1.9 & II \\
\hline 12 & 29 & $\mathrm{M}$ & PRO & 0.6 & 2.5 & II \\
\hline 13 & 55 & M & NFPA & 0.4 & 2.7 & III \\
\hline 14 & 62 & F & PRO & 0.9 & 2.1 & II \\
\hline 15 & 46 & $\mathrm{M}$ & PRO & 0.1 & 0.9 & I \\
\hline 16 & 55 & $\mathrm{~F}$ & PRO & 0.3 & 3.1 & III \\
\hline 17 & 38 & F & NFPA & 0.4 & 2.1 & II \\
\hline 18 & 31 & M & GH & 0.2 & 3.3 & III \\
\hline
\end{tabular}

M, Male; F, female; NFPA, nonfunctioning pituitary adenomas; PRO, PRO-secreting adenomas; GH, GH-secreting adenomas.

\section{Cell culture}

GH3 rat pituitary cells were cultured at $37^{\circ} \mathrm{C}$ in $35 \mathrm{~mm}$ dishes in a humidified atmosphere of $95 \%$ air and 5\% CO2. The culture medium was DMEM with $10 \%$ fetal bovine serum (FBS). Cultures were fed every other day.

\section{RNA construction and transfection}

Chemically synthesized RNA interference molecules for CDK5 were purchased from Invitrogen Trading company. The sequences used were as follows: CCGGGAAACCCAUGAGAUUTT. A nonsilencing RNA (UUCUCCGAACGUGUCACGU) duplex was used as a control. Constructs were transiently transfected into $80 \%$ confluent $\mathrm{GH} 3$ cells Lipofectamine 2000 (Invitrogen) in serum-free DMEM. At $4 \mathrm{~h}$ after transfection, the medium was replaced with DMEM with $10 \%$ fetal bovine serum (FBS).

\section{Immunofluorescence}

Normal human pituitary and pituitary adenomas tissues were washed with PBS three times, fixed 
with acetone for $10 \mathrm{~min}$, washed with PBS three times for $5 \mathrm{~min}$, permeabilized with $0.3 \%$ Triton X-100 for 30 min and washed with PBS three times for $5 \mathrm{~min}$. After blocking of nonspecific binding with $10 \%$ goat serum in PBS for $1 \mathrm{~h}$ at room temperature, the tissues were incubated with primary antibody (rabbit anti-p35 antibody 1:100, Santa Cruz Biotechnology) overnight at $4^{\circ} \mathrm{C}$, washed with PBS three times for $5 \mathrm{~min}$, incubated with fluorescein isothiocyanate (FITC)-conjugated goat anti-rabbit antibodies for $1 \mathrm{~h}$, and washed with PBS three times for $5 \mathrm{~min}$.

\section{Protein Extraction and Western Blot Analysis}

Frozen pituitary and pituitary adenomas tissues were harvested by washing with ice-cold phosphate-buffered saline (PBS) three times and then scraping in ice-cold RIPA buffer (50 mM Tris, $\mathrm{pH} 7.5$, $250 \mathrm{mM} \mathrm{NaCl}, 10 \mathrm{mM}$ EDTA, 0.5\% NP-40, $1 \mu \mathrm{g} / \mathrm{mL}$ leupeptin, $1 \mathrm{mM}$ PMSF, $4 \mathrm{mM} \mathrm{NaF})$. The homogenates were sonicated $6 \mathrm{~s}$ for three times on ice, then centrifuged at $12,000 \mathrm{~g}$ for $5 \mathrm{~min}$ at $4^{\circ} \mathrm{C}$ to yield the total protein extract in the supernatants. Concentration of protein was measured with a BCA assay kit (Pierce). Then samples $(50 \mu \mathrm{g})$ were denatured, subjected to SDS-PAGE using $12 \%$ running gels and transferred to nitrocellulose membranes. After blocking with 5\% milk powder for $1 \mathrm{~h}$ at room temperature, the membranes were incubated with primary antibody, rabbit polyclonal anti-p35 antibody (1:100; Santa Cruz Biotechnology; sc-820), monoclonal anti-Cdk5 antibody (1:1,000; Upstate; 05-364), VEGF(1:1,000; Abcam; ab46154), GAPDH(1:5,000; Sigma; G8795) overnight at $4^{\circ} \mathrm{C}$.

\section{Real-time PCR}

Total RNA was isolated using the RNA isolation kit (Roche, Switzerland) according to the manufacturer's instructions. First-strand cDNAs were generated using a M-MLV first strand kit (Invitrogen; C28025-032). Real-time PCR was performed using a LightCycler 480 (Roche Diagnostics, Basel, Switzerland) IQ SYBR Green Supermix with Platinum SYBR Green qPCR SuperMix-UDG (C11733-038 (Invitrogen; C11733-038). The primer sets used for various genes were as follows: p35, 5'-atgccgacccacactacttc-3' and $3^{\prime}$ aaaccccagggagagactgt $-5^{\prime}$; Cdk5, 5'- tgaggtggtcacactgtggt- $3^{\prime}$ and $3^{\prime}$ - ctggtcatcgacatcattgc-5'; VEGF, 5'cccactgaggagtccaacat-3' and $3^{\prime}-$ tttcttgcgctttcgttttt -5 . All samples were subjected to RT-PCR along with the housekeeping gene GAPDH, having the following primer sequences: GAPDH, 5'- gagtcaacggatttggtcgt- $3^{\prime}$ and $3^{\prime}$ - ttgattttggagggatctcg-5'. The PCR conditions were as follows: $95{ }^{\circ} \mathrm{C}$ for $10 \mathrm{~min}$, followed by 40 cycles of $\left(95^{\circ} \mathrm{C}\right.$ for $15 \mathrm{~s}, 60^{\circ} \mathrm{C}$ for $30 \mathrm{~s}$ and $72{ }^{\circ} \mathrm{C}$ for $30 \mathrm{~s}$ ) and a final extension at $72{ }^{\circ} \mathrm{C}$ for $10 \mathrm{~min}$.
Melting curves were examined for the quality of the PCR amplification of each sample, and quantification was performed using the comparative CT $\left(2^{-\Delta \Delta C T}\right)$ method.

\section{ELISA}

VEGF protein levels were determined using a rat VEGF ELISA kit from R\&D Systems according to the manufacturer's instructions. In brief, GH3 cell lines at $48 \mathrm{~h}$ after treatment with short interfering RNA or Roscovitine were harvested. Total protein content of the cells was determined for standardization of VEGF production with BCA protein assay kit (Pierce Biotechnology). The culture supernatants were collected and normalized to the cell numbers. All experiments were performed in triplicates.

\section{Statistical Analysis}

Experimental data are reported as mean \pm SEM (standard error of measurement) of three independent experiments, or more, as indicated in the respective figure legends and methods. Data were analyzed with the two-tailed Student's t-test and one-way ANOVA followed by Newman-Keuls test.

\section{Results}

\section{CDK5 and p35 are expressed in normal human pituitary}

CDK5 is often described as a nervous-system specific kinase $(4,27)$, but it is also expressed in cancer cells $(8,12,13)$. In addition, CDK5 is expressed in rat anterior pituitary. However, it was unknown whether CDK5 was present and active in normal human pituitary.

We investigated and confirmed CDK5 and p35 protein expression in normal human pituitary glands obtained from autopsies of eight individuals. The anti-p35 and anti-CDK5 antibodies gave single bands in western blot analysis (Fig.1A). The p35/GAPDH ratio was used as a measure of p35 protein levels (Fig.1B). In addition, we determined p35 distribution in normal human pituitary by immunofluorescent staining. The protein was detected in about $10-20 \%$ of the pituitary cells, and the immunofluorescent signal was granular and found in the cytoplasm (Fig 2A). To further identify whether p35 and VEGF were co-localization in human normal pituitary, we performed double immunofluorescent staining of p35 and VEGF. The results showed that some VEGF (red) was partially located in p35 (green)-positive cells (Fig. 2C). The specificity of the p35 antibody was confirmed by observing the lack of immunofluorescent signal when the samples were incubated with a mixture of antibody and a blocking p35 peptide (data not shown). Our results indicated that both $\mathrm{CDK} 5$ and its activator p35 
were expressed in normal human pituitary and suggested that p35 and VEGF were co-localizated in human normal pituitary.

\section{Expression of p35 is upregulated in human pituitary adenomas}

We then asked whether the expression of CDK5 or p35 was altered in pituitary adenomas. We measured CDK5 and p35 protein expression levels by western blot analysis of human pituitary tumors (Fig. 1A). Expression of p35 was markedly higher in PRL-secreting adenomas $(n=7)$ and NFPA (nonfunctioning pituitary adenomas, $\mathrm{n}=8$ ), than in the normal glands, whereas no significant difference was detected in CDK5 levels.

A

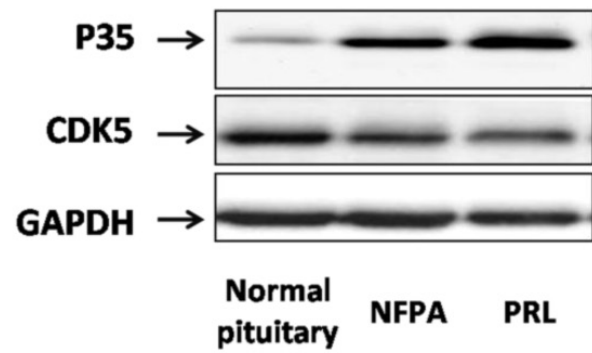

To confirm the upregulation of p35 in pituitary adenomas, we used immunofluorescent staining. As shown in Fig. 2, p35 staining was detected in about $40-50 \%$ of cells from pituitary adenomas, whereas only $10-20 \%$ of cells from normal pituitary were stained). No distinct difference in the amount of p35 staining was found between nonfunctioning pituitary adenomas, PRL-secreting adenomas and $\mathrm{GH}$-secreting adenomas (data not shown). Moreover, p35 was localized only in the cytoplasm of normal pituitary cells (Fig. 2A), whereas it was detected not only in the cytoplasm but also associated with perinuclear membranes in the cells of pituitary adenomas (Fig 2B).

B

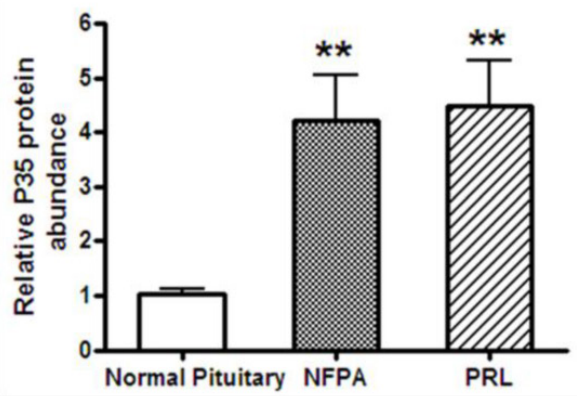

Figure I. A, Representative Western blots of P35/Cdk5 in normal human pituitary, non functional pituitary adenomas (NFPA) and PRL-secreting pituitary adenomas. Blots were reprobed with anti- GAPDH antibody to ensure equal loading; B, Ratio of $\mathrm{p} 35$ vs. GAPDH was determined by densitometry. $* \mathrm{P}<0.05$, as compared with normal pituitary group (one-way ANOVA followed by Newman-Keuls test).

$\mathbf{A}$

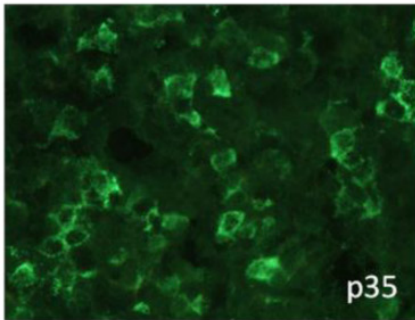

B

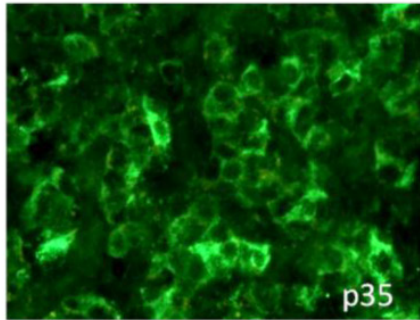

C
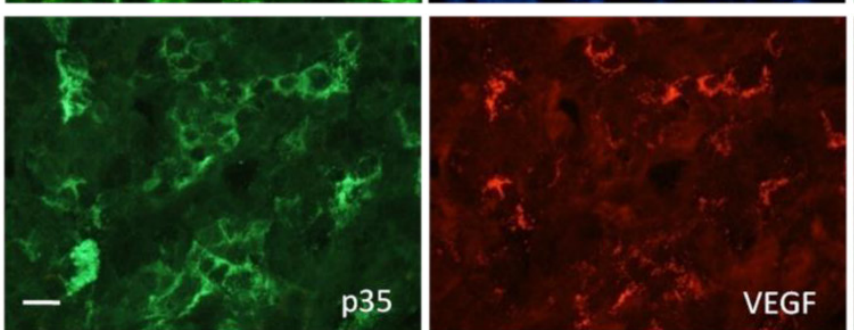
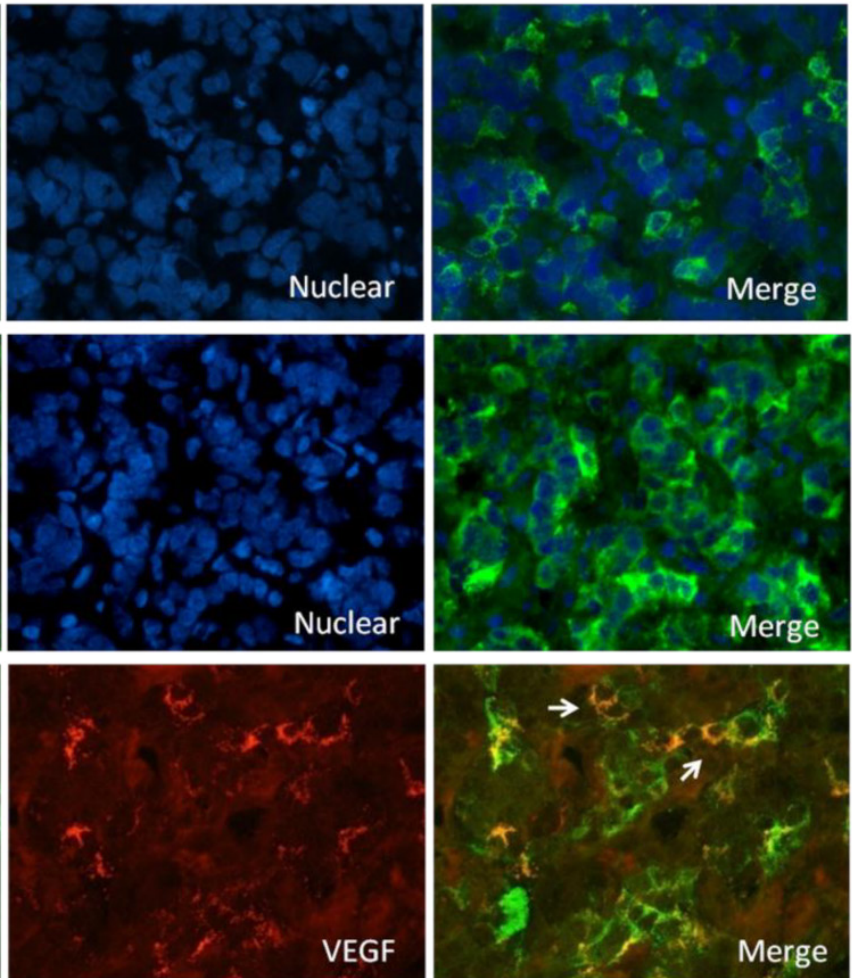

Figure 2. A, Presence of p35 in normal human pituitary. Immunofluorescence double-staining for p35 (green) and nuclear (blue) was performed; B, Presence of P35 in pituitary adenomas. Immunofluorescence double-staining for P35 (green) and nuclear (blue) was performed; C, Colocalization of p35 and and VEGF in the normal human pituitary gland. Immunofluorescence double-staining for p35 (green) and VEGF (red) was performed. Colocalization of both is indicated by filled arrowheads. Data are representative of at least three different human adult pituitary samples and at least three independent experiments. Scale bar, $20 \mu \mathrm{m}$. 

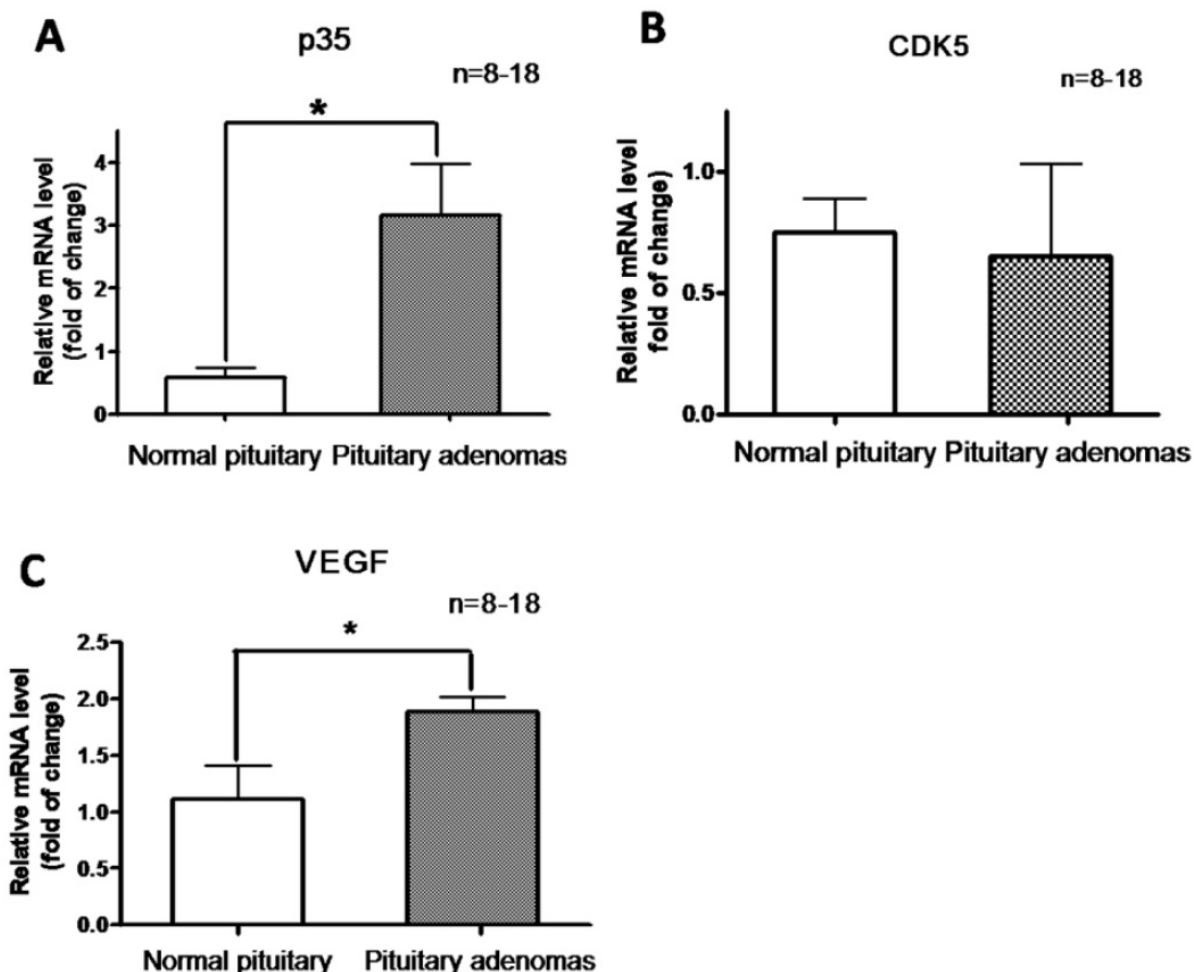

Figure 3. Relative mRNA expression of $\mathrm{P} 35$ (A), CDK5 (B) and VEGF (C) in normal human pituitary and pituitary adenomas. *P<0.05, indicates significant difference among the groups (one-way ANOVA followed by Newman-Keuls test).

In addition, we determined CDK5 and p35 mRNA levels by RT-PCR analysis of normal pituitary glands and pituitary tumors. Levels of p35 mRNA were significantly higher in pituitary adenomas than in the normal glands (Fig. 3A), whereas no significant difference in CDK5 mRNA levels was observed (Fig. 3B). These findings showed that p35 was upregulated in pituitary adenomas, suggesting an increased CDK5 activity.

\section{VEGF is upregulated in human pituitary adenomas}

We hypothesized that CDK5 might have a role in the pathogenesis of pituitary tumors by affecting VEGF expression. To explore this hypothesis, we evaluated VEGF mRNA levels by RT-PCR in the same samples used for our previous experiments, and found that pituitary adenomas showed higher VEGF mRNA levels than normal pituitary glands (Fig. 3C). In addition, we investigated VEGF protein expression by western blot in three types of pituitary adenomas (normal human pituitary, $\mathrm{n}=8$; nonfunctioning pituitary adenomas, $n=8$; PRL-secreting adenomas, $n=7$; $\mathrm{GH}$-secreting adenomas, $\mathrm{n}=3$ ) and found that the protein levels were higher in tumors than in normal glands (Fig. 4A, B). Therefore, pituitary adenomas show upregulation not only of p35 (which probably leads to higher CDK5 activity) but also of VEGF.

\section{CDK5 inhibition suppresses VEGF expression}

To explore the possible link between CDK5-p35 and VEGF in pituitary adenomas, we incubated rat pituitary GH3 cells with either DMSO or different concentrations of roscovitine (a CDK5 inhibitor). As shown in Fig. 5A, VEGF expression was significantly reduced after treatment with roscovitine, suggesting that CDK5 regulates VEGF expression. The involvement of Cdk5 is further supported by studies using short interfering RNA (siRNA) targeting the Cdk5 mRNA in GH3 cells. We found a significant decrease of VEGF in cells transfected with Cdk5 siRNA, compared with cells transfected with control siRNA (Fig. $5 B)$.

The level of VEGF that was released into the culture medium from GH3 cells was directly measured by ELISA method on 48 hours after treatment. Figure $5 \mathrm{C}$ represents that VEGF production of roscovitine-treated GH3 cells was significantly decreased compared to that of control group. Figure 5D represents that VEGF production of CDK5 siRNA-transfected GH3 cells also was significantly decreased compared to that of control siRNA-transfected group. The results were in line with the findings in Western blotting analysis. Taken together, our results point to a VEGF-mediated role for CDK5 in pituitary adenomas. 

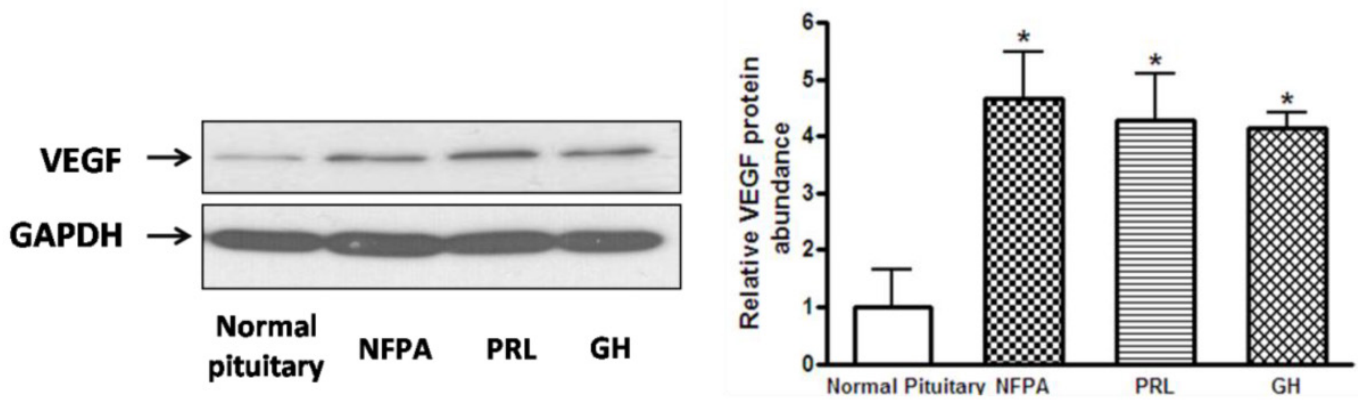

Figure 4. A, Representative Western blots of VEGF in normal human pituitary and pituitary adenomas (non functional pituitary adenomas (NFPA), PRLand GH-secreting pituitary adenomas. Blots were reprobed with anti- GAPDH antibody to ensure equal loading; B, Ratio of VEGF vs. GAPDH was determined by densitometry (Student's $t$ test was performed to determine the significance). $* \mathrm{P}<0.05$, as compared with normal pituitary group.

A

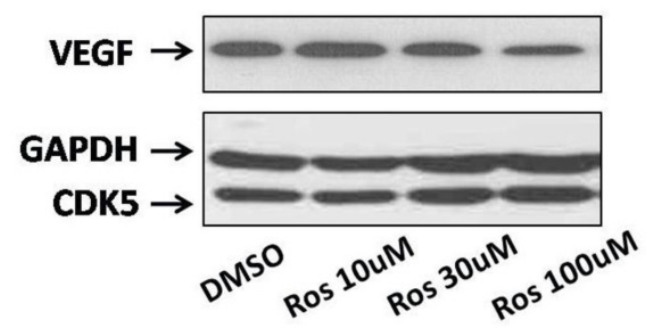

C

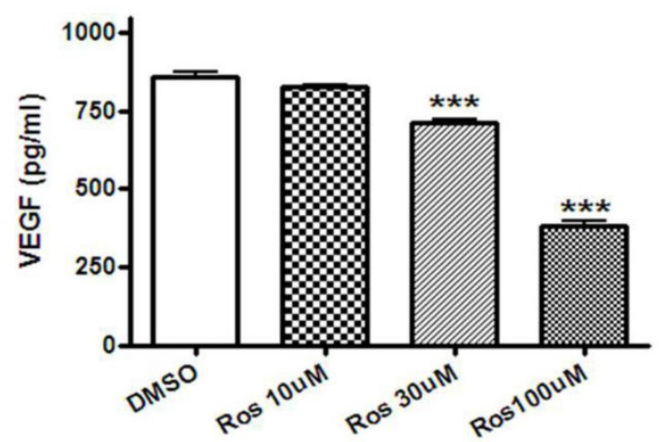

B

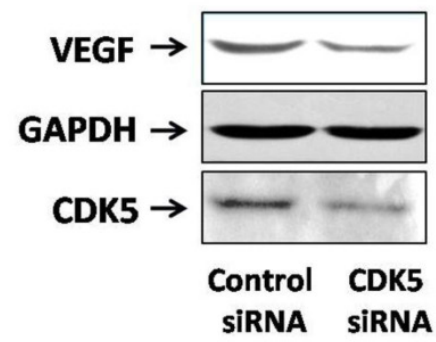

D

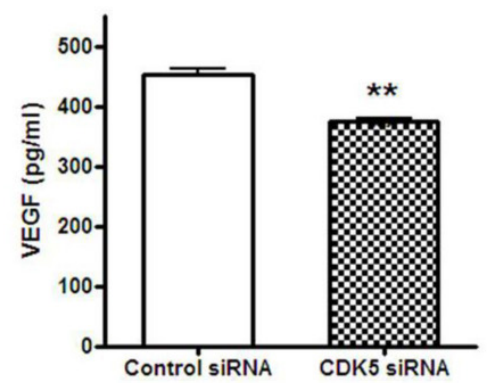

Figure 5. A, Pretreatment with roscovitine significantly decreased VEGF expression in GH3 cells. Blots were reprobed with anti- GAPDH antibody to en sure equal loading; 5B, Effect of Cdk5 siRNA on VEGF expression in GH3 cells. Blots were reprobed with anti- GAPDH antibody to en sure equal loading; $5 C$, Quantification of the level of VEGF secretion from vehicle or roscovitine-treated GH3 cells. Reduction of VEGF secretion were detected in roscovitine-treated GH3 cells compared with vehicle group; 5D, Quantification of the level of VEGF secretion from control siRNA or CDK5 siRNA-transfected GH3 cells. Reduction of VEGF secretion were detected in CDK5 siRNA-transfected GH3 cells compared with vehicle group. VEGF secretion was quantified as by ELISA as described above. Data represents means \pm SEM of three independent experiments. one-way ANOVA was performed to determine the significance $(* * p<0.01$ and $* * * p<0.001)$.

\section{Discussion}

Our findings show that active CDK5, associated with p35, is present in normal pituitary, and that CDK5 activity (as indicated by p35 levels) is upregulated in pituitary adenomas without distinct diversity between different pituitary adenomas types. Furthermore, we found that inhibition of CDK5 activity suppressed VEGF expression in pituitary cells. These results suggest that $\mathrm{CDK} 5$ activity may play a role in regulating pathological processes in pituitary tumors. To the best of our knowledge, this is the first report that provides evidence for a possible function of
CDK5- p35 in human pituitary adenomas.

Although the involvement of CDK5 in regulating neuronal processes is well documented, its role in cancer cells is only beginning to be understood $(8,12$, 13). Interestingly, CDK5 regulates angiogenesis and migration of endothelial cells, suggesting that CDK5 is a new potential target for antiangiogenic therapy (11). In addition, CDK5 substrates such as amphiphysin 1, MUNC18 (also known as STXBP1 or syntaxin-binding protein 1) and PAK participate in regulating secretion $(6,7,25)$. CDK5-mediated phosphorylation of EPRS is required for posttranscriptional suppression of VEGF-A expression (2). Furthermore, in- 
hibiting or downregulating CDK5 leads to reduced activity of the small GTPase RAC1 and to disorganization of the actin cytoskeleton (1). CDK5 exerts its functions in the pituitary, in the central nervous system and in most pituitary endocrine cells. Exocytosis from several types of pituitary endocrine cell can be diminished by inhibition of CDK5 activity (29).

A proposed role for VEGF in promoting pituitary cell proliferation has been suggested by the fact that VEGF and its receptors are widely expressed in pituitary tumors, especially in non-functioning adenomas $(14,19)$. Overexpression of VEGF in the anterior pituitary might downregulate negative regulators of angiogenesis (9) and thus promote the angiogenic process in endothelial cells. Furthermore, the use of anti-VEGF antibodies reduced the growth of pituitary adenomas in a mouse model of multiple endocrine neoplasia type 1 (10). Moreover, administration of bevacizumab (a humanized monoclonal antibody blocking VEGF) may contribute to long-term stabilization of pituitary tumors (22), emphasizing the role played by VEGF in promoting pituitary tumor progression.

Inhibitors of VEGF and of VEGF receptors have been approved for cancer therapy, but their effects are temporary and often followed by tumor regrowth and progression. Therefore, there is an urgent need to identify new therapeutic targets to inhibit vessel growth by interfering not with the response to a single growth factor, but with other steps of the angiogenic cascade. Here, we have shown that VEGF (which is prevalent in the pituitary and critical for the process of vasculogenesis and angiogenesis) is regulated by CDK5 and that its levels can be reduced by treatment with the CDK5 inhibitor roscovitine. At the concentration used in our experiments, roscovitine would also inhibit CDK1 and CDK2 (16); however, CDK5 is the most likely target of roscovitine because CDK2 levels are low in the anterior pituitary and CDK1 is not expressed in corticotropes (data not shown). Our results suggest that roscovitine and other CDK5 inhibitors have potential as antiangiogenic agents and might be useful for treatment of pathological processes characterized by excessive angiogenesis. The effects of roscovitine could be doubly beneficial, by inhibiting both metastasis and angiogenesis $(2,11)$.

The regulatory functions of CDK5 in pituitary adenomas should be further investigated. CDK5 is a proline-directed serine/threonine kinase. In addition to an absolute requirement for proline in the +1 position, CDK5 shows a marked preference for a basic residue in the +3 position. Its consensus phosphorylation sequence is $(\mathrm{S} / \mathrm{T}) \mathrm{PX}(\mathrm{K} / \mathrm{H} / \mathrm{R})$, where $X$ can be any amino acid $(3,26)$. Sequence analysis of VEGF receptors (data not shown) indicates that only VEGF receptor 2 (KDR) seems to have a putative CDK5 target site. Onofri et al. reported that KDR was expressed in vessel endothelial cells in normal and adenomatous pituitaries, and partially CD31 (angiogenesis marker)-positive vessels expressed KDR also (21). This result is consistent with our finding that the co-localization of KDR and CD31 in normal pituitary. Future research should investigate whether CDK5 can phosphorylate KDR.

In conclusion, our results suggest a function for CDK5 in pituitary tumors, elucidate a potential mechanism of action (through VEGF) and highlight CDK5 as a promising target for developing new therapies against pituitary tumors.

\section{Acknowledgments}

This work was supported by grants from the National Natural Science Foundation of China (31200796, 30672156), Beijing Nova Program (Z131102000413024).

\section{Competing Interests}

The authors have declared that no competing interest exists.

\section{References}

1. Alexander K, Yang HS, Hinds PW. Cellular senescence requires CDK5 repression of Rac1 activity. Mol Cell Biol. 2004; 24: 2808-2819.

2. Arif A, Jia J, Moodt RA, DiCorleto PE, Fox PL. Phosphorylation of glutamyl-prolyl tRNA synthetase by cyclin-dependent kinase 5 dictates transcriptselective translational control. Proc Natl Acad Sci. 2011; 108: 1415-1420.

3. Beaudette KN, Lew J, Wang JH. Substrate specificity characterization of a cdc2-like protein kinase purified from bovine brain. J Biol Chem. 1993; 268: 20825-20830.

4. Dhavan R, Tsai LH. A decade of CDK5. Nat Rev Mol Cell Biol. 2001; 2: 749-759.

5. Ferrara N, Bunting S. Vascular endothelial growth factor, a specific regulator of angiogenesis. Curr Opin Nephrol Hypertens. 1996; 5: 35-44.

6. Floyd S, Porro EB, Slepnev VI. Camilli P. Amphiphysin 1 binds the cyclin-dependent kinase (cdk) 5 regulatory subunit P35 and is phosphorylated by cdk5 and cdc2. J Biol Chem. 2003; 276: 8104-8110.

7. Gohla A, Bokoch GM. 14-3-3 regulates actin dynamics by stabilizing phosphorylated cofilin. Curr Biol. 2002; 12: 1704-1710.

8. Goodyear S, Sharma MC. Roscovitine regulates invasive breast cancer cell (MDA-MB231) proliferation and survival through cell cycle regulatory protein cdk5. Exp Mol Pathol. 2007; 82: 25-32.

9. Hanahan D, Folkman J. Patterns and emerging mechanisms of the angiogenic switch during tumorigenesis. Cell. 1996; 86: 353-364.

10. Korsisaari N, Ross J, Wu X. Blocking vascular endothelial growth factor-a inhibits the growth of pituitary adenomas and lowers serum prolactin level in a mouse model of multiple endocrine neoplasia type 1. Clin Cancer Res. 2008; 14: 249-258.

11. Liebl J, Weitensteiner SB, Vereb G. Cyclin-dependent kinase 5 regulates endothelial cell migration and angiogenesis. J Biol Chem. 2010; 285: 35932-35943.

12. Lin $\mathrm{H}$, Chen MC, Chiu CY. Cdk5 regulates STAT3 activation and cell proliferation in medullary thyroid carcinoma cells. J Biol Chem. 2007; 282: 2776-2784.

13. Liu R, Tian B, Gearing M. Cdk5-mediated regulation of the PIKE-A-Akt pathway and glioblastoma cell invasion. Proc Natl Acad Sci. 2008; 105: 7570-7575.

14. Lloyd RV, Scheithauer BW, Kuroki T, Vidal S, Kovacs K, Stefaneanu L. Vascular Endothelial Growth Factor (VEGF) Expression in Human Pituitary Adenomas and Carcinomas. Endocr Pathol. 1999; 10: 229-235.

15. Lohrer P, Gloddek J, Hopfner U, Losa M, Uhl E, Pagotto U, Stalla GK, Renner $\mathrm{U}$. Vascular endothelial growth factor production and regulation in rodent and human pituitary tumour cells in vitro. Neuroendocrinology. 2001; 74: 95-105.

16. Meijer L, Raymond E. Roscovitine and other purines as kinase inhibitors: From starfish oocytes to clinical trials. Acc Chem Res. 2003; 36: 417-425.

17. Melmed S. Pathogenesis of pituitary tumors. Nature Reviews in Endocrinology. 2011; 7: 257-266 
18. Melmed S. Update in pituitary disease. J Clin Endocrinol Metab. 2008; 93: 331-338

19. Nishikawa R, Cheng SY, Nagashima R, Huang HJ, Cavenee WK, Matsutani M. Expression of vascular endothelial growth factor in human brain tumours. Acta Neuropathol (Berl). 1998; 96: 453-462.

20. Niveiro M, Aranda FI, Peiro' G, Alenda C, Picó A. Immunohistochemical analysis of tumor angiogenic factors in human pituitary adenomas. Hum Pathol. 2005; 36: 1090-1095.

21. Onofri C, Theodoropoulou M, Losa M, Uhl E, Lange M, Arzt E, Stalla GK, Renner U. Localization of vascular endothelial growth factor (VEGF) receptors in normal and adenomatous pituitaries: detection of a non-endothelial function of VEGF in pituitary tumours. J Endocrinol. 2006; 191: 249-261.

22. Ortiz LD, Syro LV, Scheithauer BW. Anti-VEGF therapy in pituitary carcinoma. Pituitary. 2012; 15: 445-449.

23. Seilicovich A, Pisera D, Sciascia SA, Candolfi M, Puntel M, Xiong W, Jaita G, Castro MG. Gene therapy for pituitary tumors. Curr Gene Ther. 2005; 5: 559-572.

24. Shan B, Gerez J, Haedo M, Fuertes M, Theodoropoulou M, Buchfelder M, Losa M., Stalla GK, Arzt E, Renner U. RSUME is implicated in HIF-1-induced VEGF-A production in pituitary tumour cells. Endocrine Related Cancer. 2012; 19: 13-27.

25. Shuang R, Zhang L, Fletcher A. Regulation of Munc-18/syntaxin 1A interaction by cyclin-dependent kinase 5 in nerve endings. J Biol Chem. 1998; 273: 4957-4966.

26. Songyang Z, Lu KP, Kwon YT. A structural basis for substrate specificities of protein Ser/Thr kinases: primary sequence preference of casein kinases I and II, NIMA, phosphorylase kinase, calmodulin-dependent kinase II, CDK5, and erk1. Mol Cell Biol. 1996; 16: 6486-6493.

27. Tomizawa K, Ohta J, Matsushita M. Cdk5/P35 regulates neurotransmitter release through phosphorylation and downregulation of $\mathrm{P} / \mathrm{Q}$-type voltage-dependent calcium channel activity. J Neurosci. 2002; 22: 2590-2597.

28. Torre NG, Turner HE, Wass JA. Angiogenesis in prolactinomas: regulation and relationship with tumour behaviour. Pituitary. 2005; 8: 17-23.

29. Xin X, Ferraro F, Bäck N. Eipper BA, Mains RE. Cdk5 and Trio modulate endocrine cell exocytosis. J Cell Sci. 2004; 15: 4739-4748.

30. Zhang X, Horwitz GA, Heaney AP, Nakashima M, Prezant TR, Bronstein MD, Melmed S. Pituitary tumor transforming gene (PTTG) expression in pituitary adenomas. J Clin Endocrinol Metab. 1999; 84: 761-767. 